\title{
Downregulation of microRNA-96-5p protects TM3 cells against zearalenone toxicity via targeting ATG9A
}

\author{
XIAOYUAN XU ${ }^{1}$, XIAOHUA XU ${ }^{2}$, YANLUAN ZHENG ${ }^{3}$ and LAN XU ${ }^{1}$ \\ ${ }^{1}$ Department of Obstetrics and Gynecology, The First Affiliated Hospital of Shantou University Medical College, \\ Shantou, Guangdong 515041; ${ }^{2}$ Department of Cardiology, The First People's Hospital of Jingdezhen, \\ Jingdezhen, Jiangxi 333000; ${ }^{3}$ Department of Laboratory, The First Affiliated Hospital of \\ Shantou University Medical College, Shantou, Guangdong 515041, P.R. China
}

Received November 12, 2020; Accepted April 26, 2021

DOI: $10.3892 / \mathrm{etm} .2021 .10643$

\begin{abstract}
Male infertility factor accounts for $\sim 50 \%$ of all infertility cases, and traditional treatments for male infertility are limited. The association between the dysfunction of Leydig cells and hypospermatogenesis is essential for developing novel treatment methods for male infertility. It was previously stated that elevated expression of microRNA (miR)-96-5p was associated with the toxicological response of Leydig cells to treatment with zearalenone (ZEN). However, the exact role of miR-96-5p in Leydig cells remains to be illustrated. The mouse Leydig cell line TM3 was used in the present study to investigate the role of miR-96-5p. ZEN was used to induce cell injury in TM3 cells. Cell Counting Kit-8 assay and the Ki67 staining method were used to evaluate cell viability. Reverse transcription-quantitative PCR was used to determine the expression levels of miR-96-5p. In addition, a dual luciferase assay was used to investigate the target of miR-96-5p. Annexin V/propidium iodide staining was performed to detect cell apoptosis. Western blot analysis was used to detect the expression levels of certain proteins. Finally, monodansylcadaverine (MDC) and LC3 staining were applied for monitoring the level of autophagy. ZEN inhibited the proliferation of TM3 cells in a dose-dependent manner. In addition, the level of miR-96-5p were significantly increased in ZEN-treated TM3 cells. Meanwhile, inhibition of miR-96-5p could reverse ZEN-induced decrease in viability in TM3 cells. Moreover, ZEN notably inhibited autophagy in TM3 cells and this phenomenon was reversed by the application of the miR-96-5p inhibitor. Autophagy related 9A (ATG9A) was identified as the biological target of miR-96-5p. The results derived from MDC and LC3 staining demonstrated that
\end{abstract}

Correspondence to: Dr Xiaoyuan Xu or Dr Lan Xu, Department of Obstetrics and Gynecology, The First Affiliated Hospital of Shantou University Medical College, 57 Changping Road, Shantou, Guangdong 515041, P.R. China

E-mail: g_xyxu@126.com or 2622083621@qq.com

Key words: zearalenone, microRNA-96-5p, Leydig cells, TM3 cells, ATG9A downregulation of miR-96-5p expression levels protected TM3 cells against ZEN toxicity by regulating autophagy. Inhibition of miR-96-5p expression protected TM3 cells against $\mathrm{ZEN}$ via targeting ATG9A. Therefore, miR-96-5p may serve as a potential biomarker for male infertility.

\section{Introduction}

According to the World Health Organization, the failure to achieve a clinical pregnancy after 1 year or more of regular unprotected sexual intercourse is defined as infertility (1). So far, $\sim 10-15 \%$ of couples experience infertility worldwide (2). Among all these infertility cases, the male infertility factor accounts for $\sim 50 \%$ (3). It is well known that traditional treatments of male infertility include in vitro fertilization and intrauterine insemination (3). However, intrauterine insemination only achieves modest outcomes (3) and in vitro fertilization with intracytoplasmic sperm injection is invasive and expensive. In addition, both of the traditional treatments are inefficient in treating specific causes of infertility (3). Therefore, additional research studies have to be conducted on novel treatment strategies specifically targeting the causes of male infertility.

Leydig cells are a group of cells found in clusters that are located in the interstitial space of the testes between seminiferous tubules (4). Leydig cells produce testosterone and their dysfunction causes seminiferous tubule dysfunction and fall in testosterone levels, which are common features of infertile men (4). The association between Leydig cell insufficiency and hypospermatogenesis in humans has already been confirmed (4). In addition, specific toxins that affect the function and morphology of Leydig cells may result in male infertility (5). Therefore, protecting Leydig cells from toxin-induced dysfunction may aid the development of treatment strategies for male infertility.

Mycotoxins and pesticides are typical endocrine-disrupting chemicals, which can cause poor sperm quality (5).Zearalenone $(\mathrm{ZEN})$ is a non-steroidal estrogenic mycotoxin, which is a frequent contaminant of cereal crops worldwide (6). The toxic effects of ZEN on the reproductive system and its associated reproductive disorders have been previously shown (7).

MicroRNAs (miRNAs/miRs) are a family of short non-coding RNA molecules that are $\sim 18-23$ nucleotides in 
length (8). miRNAs regulate gene expression at the post-transcriptional level by binding to the 3 '-untranslated region (3'-UTR) of their target mRNAs and inducing translational repression. As a class of small non-coding RNAs, miRNA play an essential role in the process of spermatogenesis $(8,9)$. Several miRNAs, including miR-96-5p, miR-19a-3p and miR-210-5p, were found to be associated with ZEN-induced toxicity noted in Leydig cells (10). Among these miRNAs, miR-96-5p exhibited significantly increased expression levels (10). Moreover, miR-96-5p expression was associated with cell proliferation effectors, such as Foxo1, AKT2 and PTEN (10). Therefore, the present study aimed to further investigate the role of miR-96-5p on protecting Leydig cells against ZEN-induced toxicity.

\section{Materials and methods}

Cell culture and reagents. TM3 (mouse Leydig cells) were obtained from the American Type Culture Collection and cultured in DMEM/F12 media (Gibco; Thermo Fisher Scientific, Inc.) supplemented with $10 \%$ fetal bovine serum (Gibco; Thermo Fisher Scientific, Inc.), $100 \mathrm{U} / \mathrm{ml}$ penicillin and $100 \mu \mathrm{g} / \mathrm{ml}$ streptomycin in a humidified incubator containing $5 \% \mathrm{CO}_{2}$ at $37^{\circ} \mathrm{C}$. TM3 cells were cultured overnight to allow adherence prior to treatment with ZEN (50 $\mu \mathrm{M}$; Sigma-Aldrich; Merck KGaA) for $48 \mathrm{~h}$ at $37^{\circ} \mathrm{C}$. 3-Methyladenine (3MA) was purchased from Sigma-Aldrich; Merck KGaA and used at a concentration of $5 \mathrm{mM}$.

Cell Counting Kit-8 (CCK-8) assay. The CCK-8 assay kit (Dojindo Molecular Technologies, Inc.) was used to evaluate cell viability. TM3 cells were seeded into 96 -well plates at a density of $5 \times 10^{4}$ cells/well overnight. Following treatment with certain concentrations of $\operatorname{ZEN}(0,10,25,50$ and $75 \mu \mathrm{M})$ at $37^{\circ} \mathrm{C}$ for $48 \mathrm{~h}$, the cells were incubated with $10 \mu \mathrm{l} \mathrm{CCK}-8$ solution for an additional $2 \mathrm{~h}$ at $37^{\circ} \mathrm{C}$ prior to measuring the absorbance at $450 \mathrm{~nm}$ with a microplate reader (Bio-Rad Laboratories, Inc.). ZEN (50 $\mu \mathrm{M})$ was selected in the subsequent experiments to induce a moderate decrease in cell viability. For the combined treatment, TM3 cells were transfected with the miR-96-5p inhibitor (10 $\mathrm{nM})$ for $6 \mathrm{~h}$ using Lipofectamine $200{ }^{\circledR}$ (Invitrogen; Thermo Fisher Scientific, Inc.) followed by incubation with ZEN (50 $\mu \mathrm{M})$ for an additional $48 \mathrm{~h}$, as described previously (11). Subsequently, the CCK-8 assay was conducted following the aforementioned protocol.

Reverse transcription-quantitative PCR (RT-qPCR). Total cellular RNA was isolated from TM3 cells using TRIzol ${ }^{\circledR}$ reagent (Invitrogen; Thermo Fisher Scientific, Inc.). The extracted total RNA was reverse transcribed into cDNA using a PrimeScript RT-PCR kit (Takara Bio, Inc.) according to the manufacturer's instructions. RT-qPCR was performed to determine the levels of miR-96-5p using SYBR premix Ex Taq (Takara Bio, Inc.) following the manufacturer's instructions. The primer sequences used were as follows: miR-96-5p forward, 5'-TGGCACTAGCACATTTTTGC-3'; miR-96-5p reverse, 5'-CTCAACTGGTGTCGTGGAGTC-3'; U6 forward, 5'-CTCGCTTCGGCAGCACAT-3'; and U6 reverse, 5'-AAC GCTTCACGAATT TGCGT-3'. The relative expression levels of miR-96-5p were calculated using the comparative $2^{-\Delta \Delta \mathrm{Cq}}$ method (12). U6 was used as the internal control. The temperature conditions for amplification were as follows: Pre-incubation at $98^{\circ} \mathrm{C}$ for $3 \mathrm{~min}$, followed by 40 cycles at $95^{\circ} \mathrm{C}$ for $30 \mathrm{sec}, 56^{\circ} \mathrm{C}$ for $40 \mathrm{sec}$ and $72^{\circ} \mathrm{C}$ for $40 \mathrm{sec}$.

Cell transfection. miR-96-5p mimics (5'-UUUGGCACU AGCACAUUUUUGCU-3'), inhibitors (5'-AGCAAAAAU GUGCUAGUGCCAAA-3'), and negative control (mimic NC, 5'-UUGUACUACACAAAAGUACUG-3'; inhibitor NC, 5'-CAGUACUUUUGUGUAGUACAA-3') sequences were obtained from GenePharm, Inc. miR-96-5p mimics could mimic endogenous miR-96-5p, while miR-96-5p inhibitor (antisense single-stranded oligonucleotides for miRNA inhibition) could inhibit endogenous miR-96-5p. TM3 cells were seeded into 6 -well cell culture plates $\left(1.5 \times 10^{4}\right.$ cells/well $)$ and cultured overnight prior to transfection. Cells were transfected with $10 \mathrm{nM}$ miR-96-5p mimics, miR-96-5p inhibitor by using Lipofectamine $^{\circledR} 2000$ reagent (Invitrogen; Thermo Fisher Scientific, Inc.) as previously described (13). The efficiency of transfection was determined using RT-qPCR following $48 \mathrm{~h}$ of cell incubation.

Immunofluorescence assay. TM3 cells were transfected with miR-96-5p inhibitor $(10 \mathrm{nM})$ for $6 \mathrm{~h}$ followed by incubation with ZEN $(50 \mu \mathrm{M})$ at $37^{\circ} \mathrm{C}$ for an additional $48 \mathrm{~h}$. Following treatment, the cells were fixed in $4 \%$ paraformaldehyde for $30 \mathrm{~min}$ at $4^{\circ} \mathrm{C}$, followed by permeabilization with $0.1 \%$ Triton $\mathrm{X}-100$ (Sigma-Aldrich; Merck KGaA) at $4^{\circ} \mathrm{C}$ and blocking with $5 \%$ bovine serum album (BSA; Thermo Fisher Scientific, Inc.) for $1 \mathrm{~h}$ at room temperature. Subsequently, the cells were incubated with the primary antibodies overnight at $4^{\circ} \mathrm{C}$. The primary antibodies used were as follows: Ki67 (1:200; cat. no. ab15580; Abcam) and LC3 (1:200; cat. no. ab192890; Abcam). The following morning, the cells were washed with PBS and incubated with goat anti-rabbit secondary antibodies (1:500; cat. no. ab7090; Abcam) for $1 \mathrm{~h}$ at room temperature. Subsequently, the cells were counterstained with $10 \mathrm{mg} / \mathrm{ml}$ DAPI fluorescence for $2 \mathrm{~min}$ to detect the nuclei. The stained cells were visualized and mounted using a LSM710 confocal fluorescence microscope (Carl Zeiss AG; magnification, x200).

Monodansylcadaverine (MDC) staining. After the indicated treatment conditions, the cells were plated into a 6-well plate ( $3 \times 10^{6}$ cells/well) and cultured overnight, followed by staining with $0.05 \mathrm{mM}$ MDC (Sigma-Aldrich; Merck KGaA) at $37^{\circ} \mathrm{C}$ for $30 \mathrm{~min}$. Following washing with PBS for three times, the cells were fixed with $4 \%$ paraformaldehyde at room temperature for $10 \mathrm{~min}$ and immediately observed under a fluorescence microscope (magnification, x200).

Dual-luciferase reporter assay. miRDB (http://mirdb.org/) and TargetScan (www.targetscan.org/vert_71) were used to predict the target of miR-96-5p. The dual luciferase reporter assay was performed to verify the targeting association between miR-96-5p and ATG9A. Wild-type (WT) and mutant (MT) sequences of autophagy related 9A (ATG9A) were synthesized by Shanghai GeneChem Co., Ltd. The MT and WT 3'-UTR of ATG9A was cloned into a pMIR-REPORT plasmid (H306; Obio Technology) following the manufacturer's instructions. Subsequently, the cells were co-transfected with $0.1 \mu \mathrm{g}$ WT-ATG9A or MT-ATG9A and miR-96-5p mimics or vector 

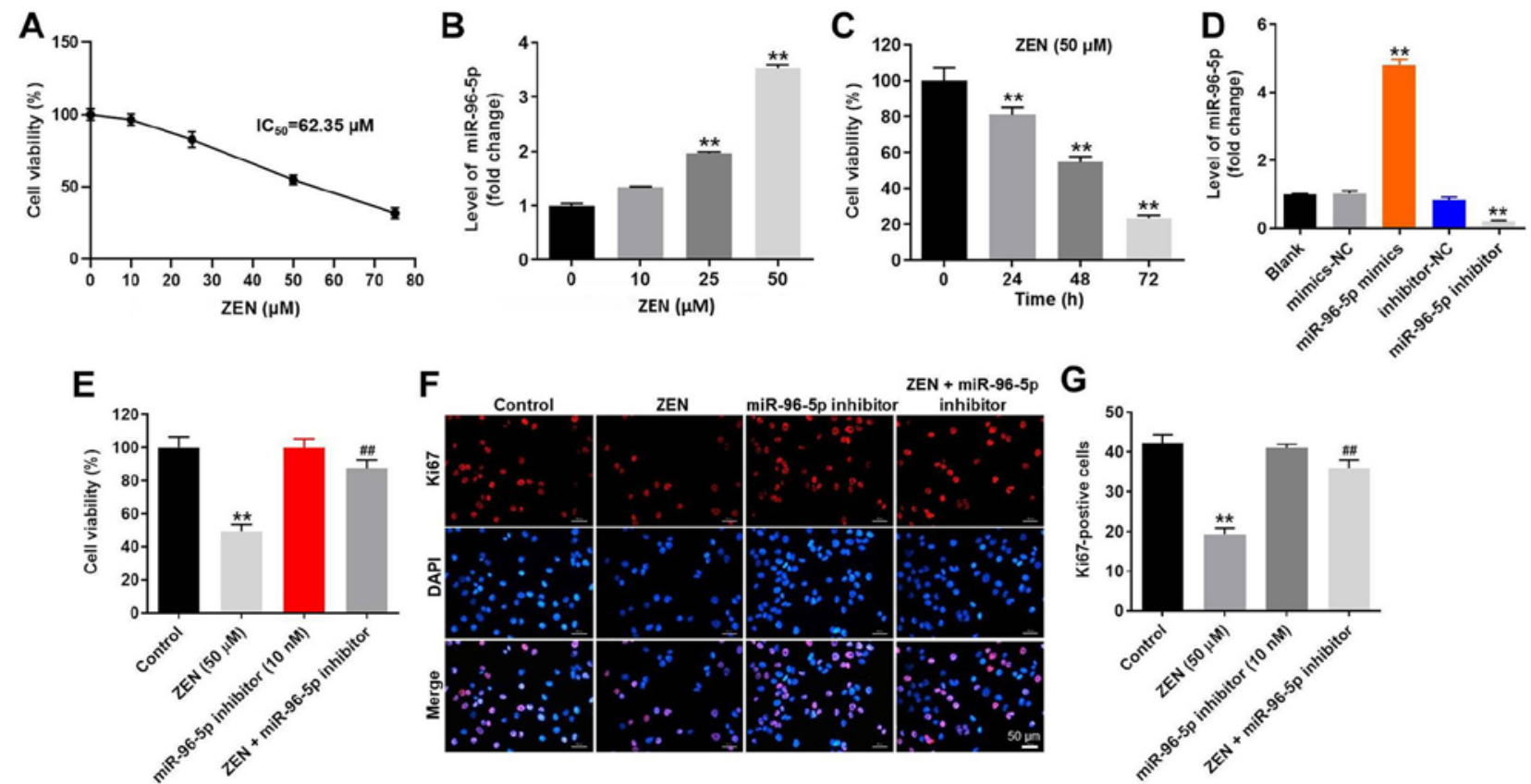

Figure 1. Downregulation of miR-96-5p reversed ZEN-induced cell viability decline in TM3 cells. (A) TM3 cells were treated with different concentrations of ZEN $(0,10,25,50$ and $75 \mu \mathrm{M})$ for $48 \mathrm{~h}$. CCK-8 assay was used to evaluate cell viability. (B) TM3 cells were treated with different concentrations of ZEN $(10,25,50$ and $75 \mu \mathrm{M})$ for $48 \mathrm{~h}$. TM3 cells treated with PBS was used as control. The level of miR-96-5p was determined by RT-qPCR. (C) TM3 cells were treated with $50 \mu \mathrm{M}$ ZEN for $0,24,48$ and $72 \mathrm{~h}$. CCK-8 assay was used to evaluate cell viability. (D) TM3 cells were transfected with NC, miR-96-5p mimics and miR-96-5p inhibitor, respectively. The level of miR-96-5p was determined by RT-qPCR. (E) The cells were treated with ZEN (50 $\mu \mathrm{M})$, miR-96-5p inhibitor $(10 \mathrm{nM})$ and ZEN + miR-96-5p inhibitor (10 nM), respectively. (F) Ki67 staining indicated cell proliferation. Magnification, x200. (G) Ki67-positive cells were counted. ${ }^{* *} \mathrm{P}<0.01$ vs. control group; ${ }^{\#} \mathrm{P}<0.01$, vs. ZEN $(50 \mu \mathrm{M})$ treated group. Control group, vehicle control. miR, microRNA; ZEN, zearalenone; CCK-8, Cell Counting Kit-8; NC, negative control; RT-qPCR, reverse transcription-quantitative PCR.

control (50 nM final concentration) using Lipofectamine 2000 (Invitrogen; Thermo Fisher Scientific, Inc.). Following $48 \mathrm{~h}$ of transfection, the Dual-Glo Luciferase Assay system (Promega Corporation) was used to measure luciferase activity according to the manufacturer's protocol. The firefly luciferase activity was normalized to the Renilla luciferase activity and presented as relative luciferase activity.

Western blot analysis. Total proteins were obtained from lysates of cultured cells using RIPA buffer (Shanghai GenePharma Co., Ltd.). The concentration levels of the proteins were measured with a BCA protein assay kit (Beyotime Institute of Biotechnology). Subsequently, total protein $(30 \mu \mathrm{g} / \mathrm{lane})$ was loaded onto SDS gels $(10 \%)$ and separated by electrophoresis. The gels were transferred to PVDF membranes. Following blocking with 5\% skimmed milk for $1 \mathrm{~h}$ at room temperature, the membrane was probed with antibodies against ATG9A (1:1,000; cat. no. ab108338; Abcam), cleaved caspase-3 (1:1,000; cat. no. ab32042; Abcam), Bcl-2 (1:1,000; cat. no. ab32124; Abcam) and beclin 1 (1:1,000; cat. no. ab210498; Abcam) overnight at $4^{\circ} \mathrm{C}$. Subsequently, the membrane was incubated with appropriate horseradish peroxidase-conjugated anti-rabbit secondary antibody (1:3,000; cat. no. ab7090; Abcam). The visualization was performed using an ECL chemiluminescent kit (Beyotime Institute of Biotechnology) according to the manufacturer's instructions. The integrated density of each band was normalized to that of the corresponding $\beta$-actin (1:1,000; cat. no. ab8226; Abcam) band. Image J software (version 2.0; National Institutes of Health) was used to quantify the intensity of the bands.
Apoptosis assay. Following the indicated treatment, cells were harvested and resuspended at a density of $5 \times 10^{5}$ cells $/ \mathrm{ml}$. Subsequently, the cells were centrifuged $(300 \mathrm{x} \mathrm{g})$ at room temperature for $5 \mathrm{~min}$, followed by washing with PBS 3 times. A total of $1 \times 10^{5}$ cells were collected in each tube. The Annexin V-FITC Apoptosis Detection kit (Thermo Fisher Scientific, Inc.) was used to quantify the percentage of apoptotic cells. The cell pellets were resuspended in $1 \mathrm{ml}$ Annexin $\mathrm{V}$ binding buffer, followed by staining with $5 \mu \mathrm{l}$ Annexin $\mathrm{V}$ and $10 \mu \mathrm{l}$ propidium iodide (PI) for $15 \mathrm{~min}$ at room temperature. Flow cytometry analysis (BD FACS Aria; BD Biosciences) was conducted within $1 \mathrm{~h}$ to detect the orange-red fluorescence of Annexin V/PI. The data was quantified by FlowJo (v7.6.5; FlowJo LLC).

Statistical analysis. All experiments were performed in triplicate. All data are presented as mean \pm SD. GraphPad Prism 7 (GraphPad Software, Inc.) was used for statistical analysis. The differences among multiple groups were analyzed using one-way ANOVA followed by Tukey's multiple comparisons test. $\mathrm{P}<0.05$ was considered to indicate a statistically significant difference.

\section{Results}

Downregulation of miR-96-5p expression reverses ZEN-induced cytotoxicity in TM3 cells. TM3 cells were cultured with various concentrations of ZEN $(0,10,25,50$ and $75 \mu \mathrm{M}$ ) for $48 \mathrm{~h}$ and the effect of this compound on cell viability was evaluated by the CCK- 8 assay. The results indicated that ZEN inhibited the proliferation of TM3 cells in a dose-dependent manner (Fig. 1A). Treatment of the cells with 
ZEN $(50 \mu \mathrm{M})$ resulted in moderate cell growth inhibition of $\sim 50 \%$ (Fig. 1A). Therefore, ZEN was used in the following experiments at a concentration of $50 \mu \mathrm{M}$. At the concentration of $75 \mu \mathrm{M}, \mathrm{ZEN}$ induced severe toxicity. It has been previously reported that miR-96-5p is closely associated with the toxicity of ZEN in Leydig cells (10). Therefore, the expression levels of miR-96-5p in ZEN-treated TM3 cells were detected by RT-qPCR. ZEN notably upregulated the expression levels of miR-96-5p in a dose-dependent manner (Fig. 1B). In addition, ZEN $(50 \mu \mathrm{M})$ markedly inhibited the viability of TM3 cells in a time-dependent manner (Fig. 1C). After $24 \mathrm{~h}$ of incubation, ZEN $(50 \mu \mathrm{M})$ resulted in moderate cell growth inhibition of $\sim 20 \%$; after $48 \mathrm{~h}$ of incubation, ZEN $(50 \mu \mathrm{M})$ resulted in moderate cell growth inhibition of $\sim 50 \%$ (Fig. 1C). Therefore, TM3 cells that were treated with ZEN $(50 \mu \mathrm{M})$ for $48 \mathrm{~h}$ were utilized in the following experiments.

In order to assess the function of miR-96-5p further, TM3 cells were transfected with miR-96-5p mimics, miR-96-5p inhibitor or NC sequences. The efficiency of transfection was evaluated using RT-qPCR and the data indicated that TM3 cells were successfully transfected with miR-96-5p mimics or inhibitor (Fig. 1D). In addition, the results of the CCK-8 assay indicated that ZEN $(50 \mu \mathrm{M})$ resulted in a significant reduction of cell viability (Fig. 1E), while the miR-96-5p inhibitor (10 nM) exhibited no significant effect on cell viability (Fig. 1D). In addition, the decrease in cell viability induced by ZEN was remarkably attenuated by the miR-96-5p inhibitor (Fig. 1E). Furthermore, the results of Ki67 staining indicated that the miR-96-5p inhibitor efficiently reversed the decrease in cell proliferation induced by ZEN in TM3 cells (Fig. 1F and G). Taken together, the results indicated that downregulation of miR-96-5p expression significantly attenuated ZEN-induced cytotoxicity in TM3 cells.

ATG9A is the target of miR-96-5p. The target of miR-96-5p was predicted using the online databases miRDB (http://www.mirdb.org/) and TargetScan (http://www. targetscan.org/vert_72/). Accordingly to previous studies, miR-96-5p was reported to have a closed association with autophagy $(11,13,14)$. Thus, the present study focused on analyzing autophagy-associated genes and found that ATG9A was the putative target of miR-96-5p (Fig. 2A). Subsequently, the association between miR-96-5p and ATG9A was verified by dual-luciferase reporter assays. The relative luciferase activity of the cells co-transfected with wild type ATG9A and miR-96-5p mimics was markedly decreased (Fig. 2B). This result demonstrated that miR-96-5p was able to bind directly to the WT 3'-UTR of ATG9A. Therefore, ATG9A was confirmed as a direct target of miR-96-5p. In addition, western blot analysis was used to validate the association between miR-96-5p and ATG9A. The expression levels of ATG9A were downregulated by miR-96-5p mimics, confirming that ATG9A was a direct target of miR-96-5p (Fig. 2C and D). Since ATG9A is a transmembrane protein and plays an essential role in autophagy (15), the induction of autophagy in TM3 cells was investigated in the subsequent experiments.

Downregulation of miR-96-5p expression protects TM3 cells from ZEN-induced apoptosis. Subsequently, the induction of TM3 cell apoptosis was detected by Annexin V/PI staining.
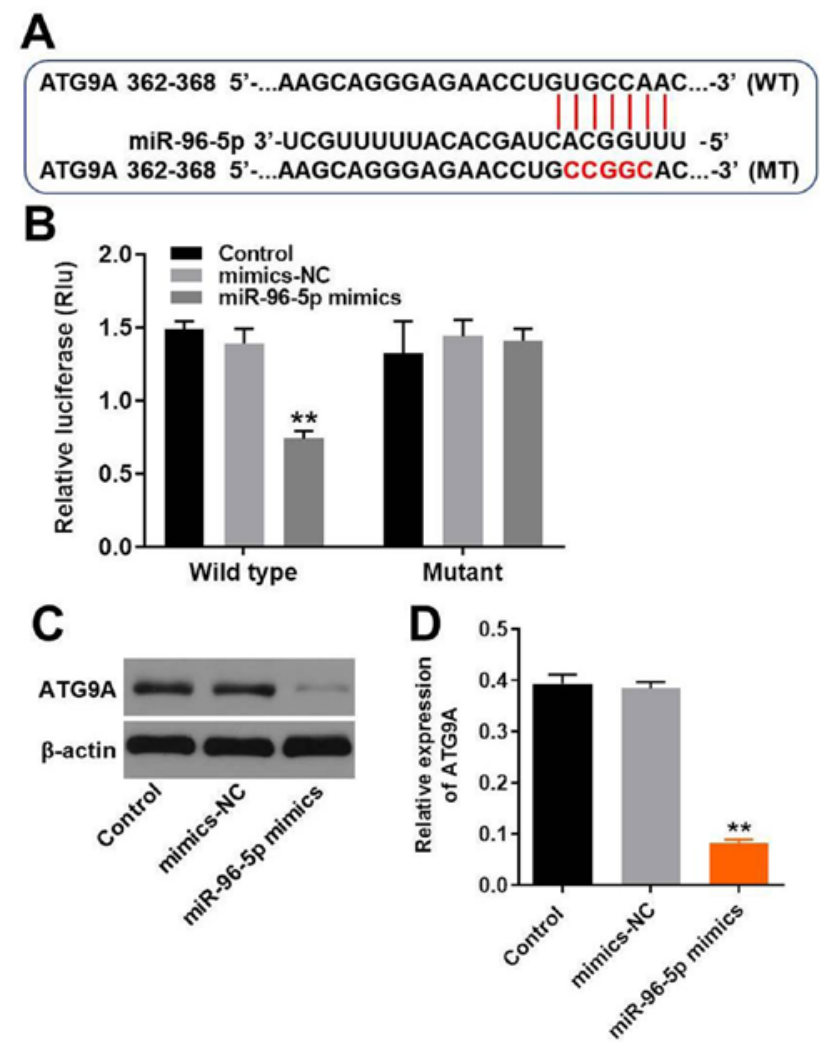

Figure 2. ATG9A is a target of miR-96-5p. (A) ATG9A was predicted as the target of miR-96-5p. (B) Dual-luciferase reporter assay validated the targeting association between miR-96-5p and ATG9A. (C) TM3 cells were transfected with mimics-NC or miR-96-5p mimics for $6 \mathrm{~h}$. At $48 \mathrm{~h}$ post-transfection, western blotting was performed to detect the expression level of ATG9A in cells.TM3 cells without transfection were used as blank control. (D) The expression of ATG9A was quantified. ${ }^{* *} \mathrm{P}<0.01$, vs. control group. Control group, vehicle control. miR, microRNA; ATG9A, autophagy related $9 \mathrm{~A} ; \mathrm{NC}$, negative control.

ZEN (50 $\mu \mathrm{M})$ induced TM3 cell apoptosis (Fig. 3A and B), whereas the miR-96-5p inhibitor had no effect on cell apoptosis (Fig. 3A and B). In addition, downregulation of miR-96-5p expression markedly decreased ZEN-induced cell apoptosis. It is interesting to note that the protective effect of the miR-96-5p inhibitor against ZEN-induced apoptosis was abolished by 3MA, which is an autophagy inhibitor. Moreover, the expression levels of the apoptosis-associated proteins (cleaved caspase-3 and Bcl-2) were evaluated by western blot analysis. The results indicated that $\mathrm{ZEN}$-induced upregulation of cleaved caspase-3 expression was reversed by miR-96-5p inhibitor transfection in the cells (Fig. 3C-E). Similarly, the ZEN-induced decrease in Bcl-2 expression levels was reversed by miR-96-5p inhibitor transfection in the cells (Fig. 3C-E). Moreover, the effects of the miR-96-5p inhibitor on cleaved caspase- 3 and $\mathrm{Bcl}-2$ expression levels were eliminated in the presence of 3MA, which was consistent with the aforementioned findings. Taken together, the results indicated that downregulation of miR-96-5p expression protected TM3 cells against ZEN-induced apoptosis by regulating autophagy.

Downregulation of miR-96-5p expression protects TM3 cells against ZEN cytotoxicity by promoting autophagy. In order to confirm the association between the miR-96-5p inhibitor 
A

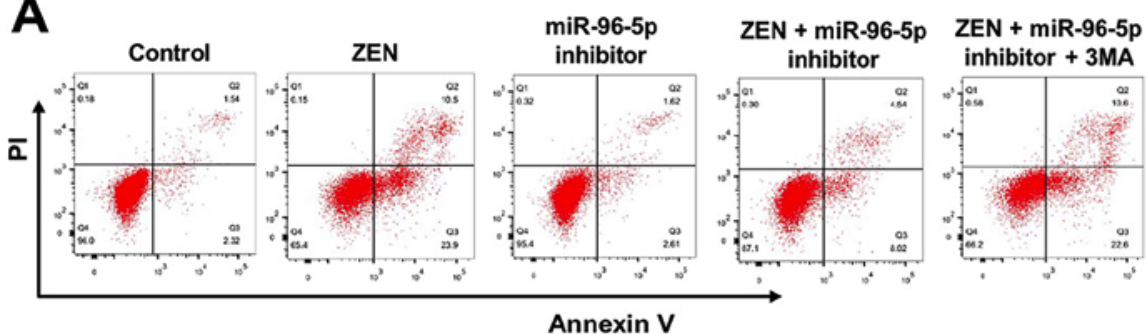

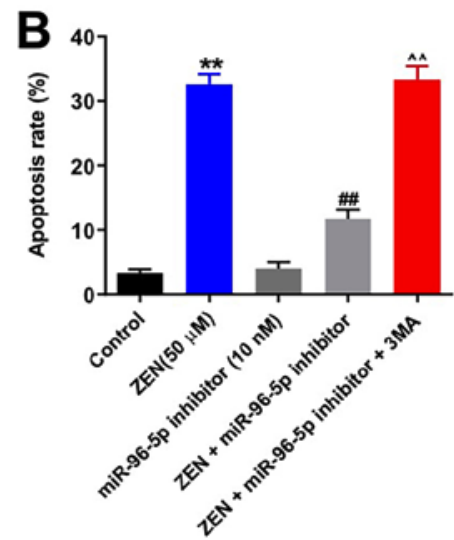

E

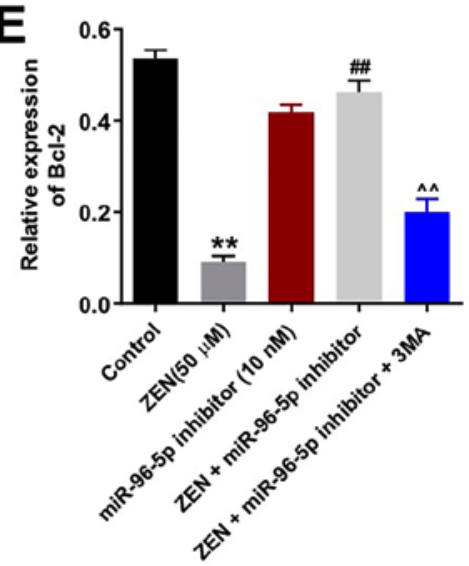

Figure 3. ZEN-induced apoptosis of TM3 is inhibited by miR-96-5p inhibitor. TM3 cells were transfected with miR-96-5p inhibitor (10 nM) for 6 h, then cells were treated with $50 \mu \mathrm{M}$ ZEN or/and $5 \mathrm{mM} 3 \mathrm{MA}$ for $48 \mathrm{~h}$. (A) Annexin V/PI staining were used to detect cell apoptosis. (B) The apoptosis rate of the cells from each group was quantified. (C) The levels of cleaved caspase 3 and Bcl-2 were estimated by western blotting. $\beta$-actin was used as an internal control. (D and E) The levels of cleaved caspase 3 and Bcl-2 were quantified. ${ }^{* *} \mathrm{P}<0.01$, vs. control; ${ }^{\# \#} \mathrm{P}<0.01$, vs. ZEN (50 $\left.\mu \mathrm{M}\right)$ group. ${ }^{\wedge} \mathrm{P}<0.01$, vs. ZEN + miR-96 inhibitor group. Control group, vehicle + inhibitor-NC. miR, microRNA; NC, negative control; ZEN, zearalenone; 3MA, 3-methyladenine.

and the induction of cell autophagy, MDC staining was performed. ZEN (50 $\mu \mathrm{M})$ decreased the number of autophagosomes in TM3 cells, whereas this effect was reversed by transfection of the cells with the miR-96-5p inhibitor (Fig. 4A and B). As expected, the autophagy-promoting effect of the miR-96-5p inhibitor was neutralized by 3MA (Fig. 4A and B). Furthermore, the expression levels of the autophagy-associated proteins (ATG9A and beclin 1) were assessed (Fig. 4C-E) (16).ZEN $(50 \mu \mathrm{M})$ significantly decreased the expression levels of ATG9A and Beclin 1 (Fig. 4C-E), while the miR-96-5p inhibitor $(10 \mathrm{nM})$ triggered the upregulation of ATG9A expression compared with the control group (Fig. 4C). Moreover, the inhibition of miR-96-5p reversed ZEN-induced decrease in ATG9A and Beclin 1 expression levels (Fig. 4C-E). Similarly, the effects of the miR-96-5p inhibitor were neutralized by 3MA (Fig. 4C-E). In addition to MDC staining, LC3 staining was used to confirm the induction of autophagy in ZEN-treated TM3 cells. ZEN-induced decline in autophagy was ameliorated by treatment of the cells with the miR-96-5p inhibitor, while the enhancement of autophagy by the miR-96-5p inhibitor was abrogated following 3MA treatment (Fig. 5A and B). Taken together, the data demonstrated that downregulation of miR-96-5p expression protected TM3 cells against ZEN cytotoxicity by promoting autophagy.

\section{Discussion}

The insufficiency and dysfunction of Leydig cells is associated with male infertility (4). Increasing sperm production or motility by optimizing testosterone production from Leydig cells is the typical medical therapy (17). In the present study, downregulation of miR-96-5p expression levels protected TM3 Leydig cells against ZEN-induced toxicity, providing a potential new biomarker for male infertility.

In the present study, the data demonstrated that downregulation of miR-96-5p expression protected TM3 cells against ZEN-induced toxicity via promoting autophagy. A previous study conducted by Yu et al (11) demonstrated that inhibition of miR-96-5p expression promoted autophagy in the human hepatic stellate cell line LX-2 via the upregulation of ATG7. Moreover, Shi et al (14) demonstrated that the overexpression of miR-96-5p was able to inhibit autophagy in the human breast cancer cell lines MCF-7 and MDA-MB-231 through downregulation of FOXO1. In addition, Ma et al (18) indicated that miR-96 could induce the autophagy in prostate cancer cells via inhibition of mTOR. The aforementioned studies indicated that miR-96-5p could regulate autophagy via the regulation of several autophagy-associated genes (such as ATG7 and mTOR) or autophagy-associated signaling molecules (such as FOXO1). The present study 

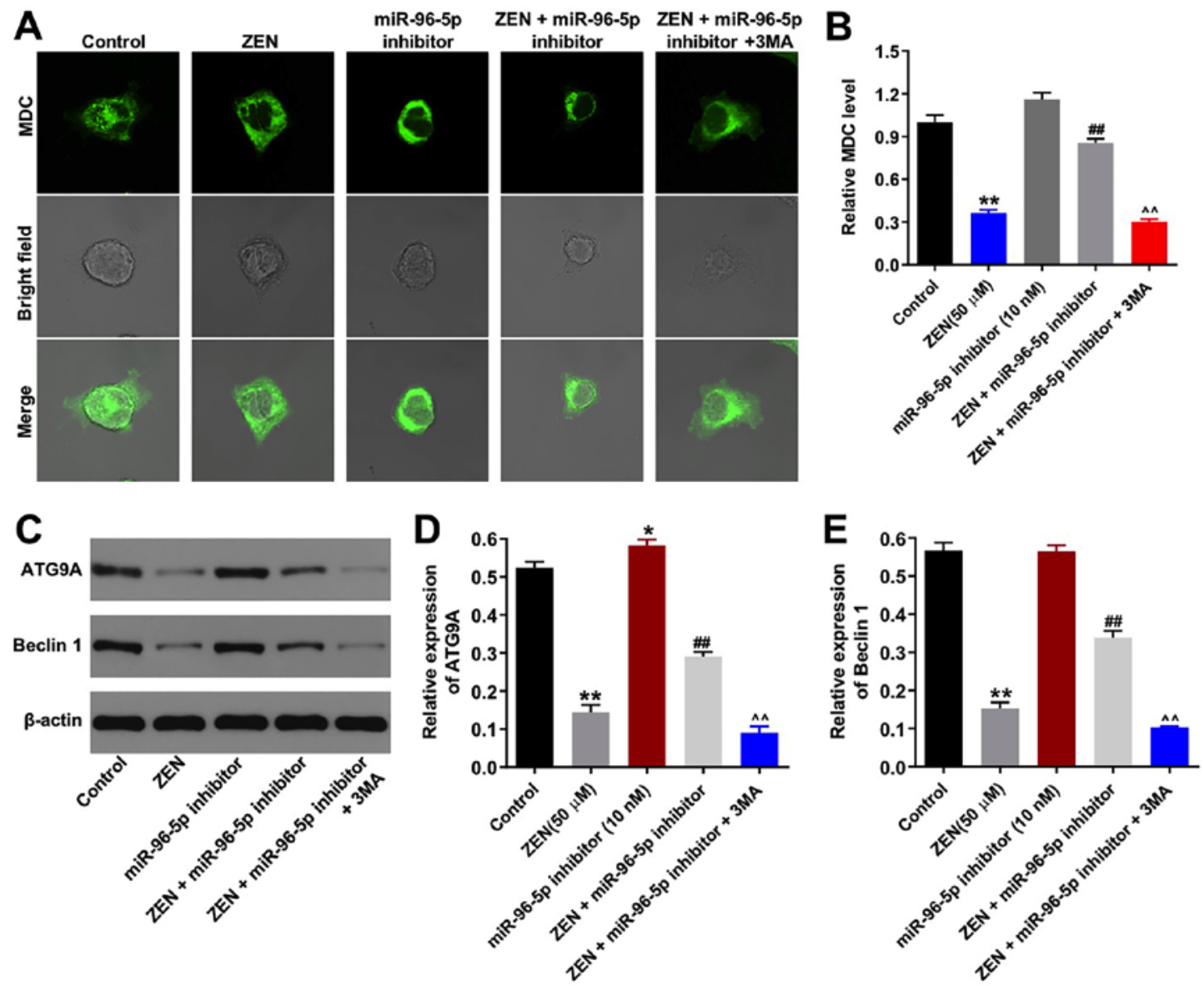

Figure 4. Downregulation of miR-96-5p protects TM3 cells against ZEN cytotoxicity via promoting autophagy. TM3 cells were transfected with miR-96-5p inhibitor $(10 \mathrm{nM})$ for $6 \mathrm{~h}$, then cells were treated with $50 \mu \mathrm{M} \mathrm{ZEN} \mathrm{and/or} 5 \mathrm{mM} 3 \mathrm{MA}$ for $48 \mathrm{~h}$. (A) MDC-stained autophagosome in TM3 cells from each group. Magnification, x400. (B) MDC-positive autophagosomes were counted. (C) The expression levels of ATG9A and beclin 1 were detected using western blotting and (D and E) quantified. ${ }^{\# \#} \mathrm{P}<0.01$, vs. ZEN (50 $\left.\mu \mathrm{M}\right)$ group. ${ }^{\wedge} \mathrm{P}<0.01$, vs. ZEN + miR-96 inhibitor group. ${ }^{*} \mathrm{P}<0.05$, ${ }^{* *} \mathrm{P}<0.01$, vs. Control group, vehicle + inhibitor-NC. miR, microRNA; NC, negative control; ZEN, zearalenone; 3MA, 3-methyladenine; MDC, monodansylcadaverine; ATG9A, autophagy related $9 \mathrm{~A}$.

A

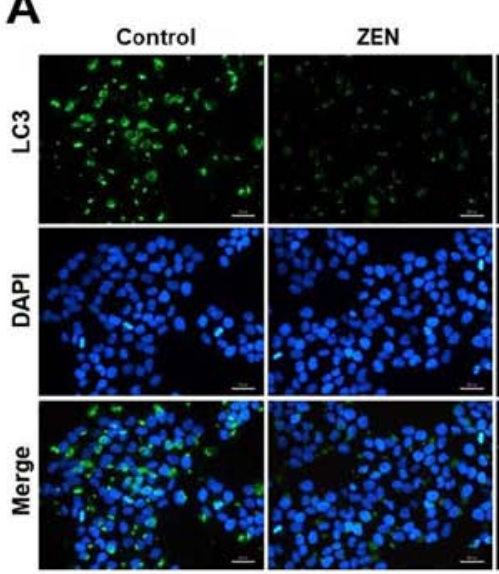

ZEN + miR-96-5p
inhibitor
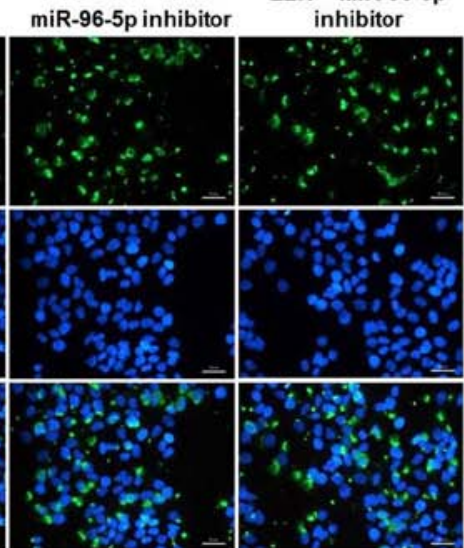

ZEN + miR-96-5p inhibitor + 3MA

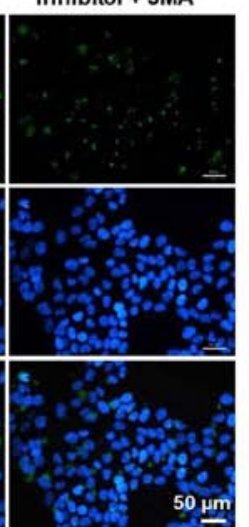

B

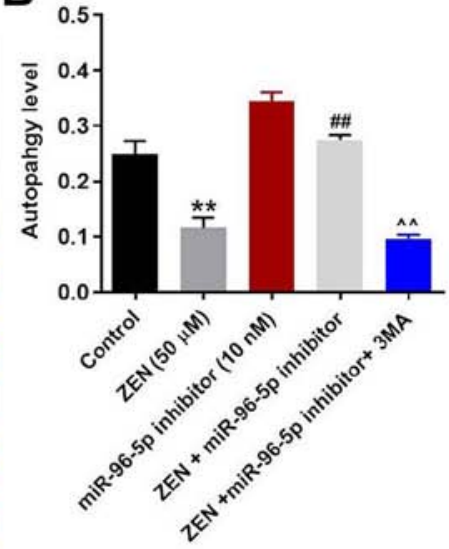

Figure 5. miR-96-5p inhibitor-induced autophagy in TM3 cells is inhibited by 3MA treatment. TM3 cells were transfected with miR-96-5p inhibitor (10 nM) for $6 \mathrm{~h}$, then cells were treated with $50 \mu \mathrm{M}$ ZEN and/or $5 \mathrm{mM} 3 \mathrm{MA}$ for $48 \mathrm{~h}$. (A) LC3-stained autophagosome in TM3 cells was detected with fluorescence microscope. Magnification, $\mathrm{x} 200$. (B) LC3-positive autophagosomes were counted. ${ }^{* *} \mathrm{P}<0.01$, vs. Control. ${ }^{\# \#} \mathrm{P}<0.01$, vs. ZEN $(50 \mu \mathrm{M})$ group. ${ }^{\wedge} \mathrm{P}<0.01$, vs. ZEN + miR-96 inhibitor group. Control group, vehicle + inhibitor-NC. miR, microRNA; NC, negative control; ZEN, zearalenone; 3MA, 3-methyladenine.

demonstrated that ATG9A was a direct binding target of miR-96-5p. ATG9A is a membrane protein, that is essential for autophagy (19). Downregulation of miR-96-5p significantly induced the autophagy of ZEN-treated TM3 cells via the upregulation of ATG9A. However, one miRNA can regulate several mRNAs, thus further studies are needed to investigate whether miR-96-5p could regulate the progression of male infertility via targeting other gene targets. 
Furthermore, the present study data found that miR-96-5p inhibitor could suppress the apoptosis of ZEN-treated TM3 cells. However, the inhibitory effects of miR-96-5p inhibitor on apoptosis in ZEN-treated TM3 cells were reversed by the treatment with 3MA. The results suggested that downregulation of miR-96-5p could inhibit apoptosis in ZEN-treated TM3 cells via inducing autophagy. In addition, the data demonstrated that the miR-96-5p inhibitor alone exhibited no influence on the induction of cell apoptosis; thus, miR-96-5p inhibitor did not regulate cell apoptosis directly. In addition, rescue experiments demonstrated that the miR-96-5p inhibitor protected TM3 cells against ZEN-induced cytotoxicity by promoting autophagy. The enhanced induction of autophagy by the miR-96-5p inhibitor may aid the maintenance of cell homeostasis and enable the cells to survive under adverse conditions, such as in the case of ZEN-induced toxicity (20).

It is interesting to note that the changes in the expression levels of cleaved caspase-3 and Bcl-2 were inconsistent (Fig. 3D and E). It was deduced that cleaved caspase-3 may be activated by another pathway in addition to the typical mitochondrial pathway of apoptosis. However, the specific pathway by which cleaved caspase- 3 was activated remained unclear.

In the present study, the data demonstrated that downregulation of miR-96-5p expression reversed $\mathrm{ZEN}$-induced cytotoxicity in TM3 cells by targeting ATG9A. When the expression of miR-96-5p was downregulated in TM3 cells, ATG9A-associated autophagy was increased. The findings suggested that miR-96-5p may serve as a potential biomarker for male infertility.

\section{Acknowledgements}

Not applicable.

\section{Funding}

This study was supported by Natural Science Foundation of Guangdong Province (grant no. 2016A030310075).

\section{Availability of data and materials}

The datasets used and/or analyzed during the current study are available from the corresponding author on reasonable request.

\section{Authors' contributions}

LX conceived and supervised the study. LX, XYX and XHX designed the study. XHX and YZ performed the experiments and analyzed the data. LX and XYX confirmed the authenticity of all the raw data. All authors reviewed the results and approved the final version of the manuscript.

\section{Ethics approval and consent to participate}

Not applicable.

\section{Patient consent for publication}

Not applicable.

\section{Competing interests}

The authors declare that they have no competing interests.

\section{References}

1. Zegers-Hochschild F, Adamson GD, de Mouzon J, Ishihara O, Mansour R, Nygren K, Sullivan E and van der Poel S; International Committee for Monitoring Assisted Reproductive Technology; World Health Organization: The International Committee For Monitoring Assisted Reproductive Technology (ICMART) and the World Health Organization (WHO) revised glossary on ART terminology, 2009. Hum Reprod 24: 2683-2687, 2009.

2. Okutman O, Rhouma MB, Benkhalifa M, Muller J and Viville S: Genetic evaluation of patients with non-syndromic male infertility. J Assist Reprod Genet 35: 1939-1951, 2018.

3. Stojanov M, Baud D, Greub G and Vulliemoz N: Male infertility: The intracellular bacterial hypothesis. New Microbes New Infect 26: 37-41, 2018.

4. Winters SJ, Moore JP Jr and Clark BJ: Leydig cell insufficiency in hypospermatogenesis: A paracrine effect of activin-inhibin signaling? Andrology 6: 262-271, 2018.

5. Eze UA, Huntriss J, Routledge MN and Gong YY: Toxicological effects of regulated mycotoxins and persistent organochloride pesticides: In vitro cytotoxic assessment of single and defined mixtures on MA-10 murine Leydig cell line. Toxicol In vitro 48: 93-103, 2018.

6. Boeira SP, Filho CB, Del'Fabbro L, Royes LF, Jessé CR, Oliveira MS and Furian AF: Possible role for glutathione-S-transferase in the oligozoospermia elicited by acute zearalenone administration in Swiss albino mice. Toxicon 60: 358-366, 2012.

7. Zhou M, Yang L, Shao M, Wang Y, Yang W, Huang L, Zhou X, Jiang S and Yang Z: Effects of zearalenone exposure on the TGF- $\beta 1 / \mathrm{Smad} 3$ signaling pathway and the expression of proliferation or apoptosis related genes of post-weaning gilts. Toxins (Basel) 10: 49, 2018.

8. Gao H, Wen H, Cao C, Dong D, Yang C, Xie S, Zhang J, Huang X, Huang X, Yuan S and Dong W: Overexpression of MicroRNA-10a in germ cells causes male infertility by targeting Rad51 in mouse and human. Front Physiol 10: 765, 2019.

9. Zhang Y, Wang Z and Gemeinhart RA: Progress in microRNA delivery. J Control Release 172: 962-974, 2013.

10. Wang M, Wu W, Li L, He J, Huang S, Chen S, Chen J, Long M, Yang S and Li P: Analysis of the miRNA expression profiles in the zearalenone-exposed TM3 leydig cell line. Int J Mol Sci 20: 635, 2019.

11. Yu K, Li N, Cheng Q, Zheng J, Zhu M, Bao S, Chen M and Shi G: miR-96-5p prevents hepatic stellate cell activation by inhibiting autophagy via ATG7. J Mol Med (Berl) 96: 65-74, 2018.

12. Livak KJ and Schmittgen TD: Analysis of relative gene expression data using real-time quantitative PCR and the 2(-Delta Delta C(T)) method. Methods 25: 402-408, 2001.

13. Mao Z, Yao M, Li Y, Fu Z, Li S, Zhang L, Zhou Z, Tang Q, Han X and Xia Y: miR-96-5p and miR-101-3p as potential intervention targets to rescue $\mathrm{TiO}_{2} \mathrm{NP}$-induced autophagy and migration impairment of human trophoblastic cells. Biomater Sci 6: 3273-3283, 2018.

14. Shi Y, Zhao Y, Shao N, Ye R, Lin Y, Zhang N, Li W, Zhang Y and Wang S: Overexpression of microRNA-96-5p inhibits autophagy and apoptosis and enhances the proliferation, migration and invasiveness of human breast cancer cells. Oncol Lett 13: 4402-4412, 2017.

15. Judith D and Tooze SA: ATG9A supplies PtdIns4P to the autophagosome initiation site. Autophagy 15: 1660-1661, 2019.

16. Nishikawa M, Miyake H, Liu B and Fujisawa M: Expression pattern of autophagy-related markers in non-metastatic clear cell renal cell carcinoma: Association with disease recurrence following radical nephrectomy. J Cancer Res Clin Oncol 141: 1585-1591, 2015.

17. Dabaja AA and Schlegel PN: Medical treatment of male infertility. Transl Androl Urol 3: 9-16, 2014.

18. Ma Y, Yang HZ, Dong BJ, Zou HB, Zhou Y, Kong XM and Huang YR: Biphasic regulation of autophagy by miR-96 in prostate cancer cells under hypoxia. Oncotarget 5: 9169-9182, 2014.

19. Kong Y, Huang T, Zhang H, Zhang Q, Ren J, Guo X, Fan H and Liu L: The lncRNA NEAT1/miR-29b/Atg9a axis regulates IGFBPrP1-induced autophagy and activation of mouse hepatic stellate cells. Life Sci 237: 116902, 2019.

20. Kasprowska-Liśkiewicz D: The cell on the edge of life and death: Crosstalk between autophagy and apoptosis. Postepy Hig Med Dosw (Online) 71: 825-841, 2017.

This work is licensed under a Creative Commons Attribution-NonCommercial-NoDerivatives 4.0 International (CC BY-NC-ND 4.0) License. 